\title{
A SURVEY OF ART LEARNING IN KINDERGARTEN DURING THE COVID 19 PANDEMIC
}

\section{Joko Pamungkas, Amir Syamsudin, Harun Ar Rosid}

Yogyakarta State University, Indonesia

Email: joko_pamungkas@uny.ac.id, amirsyamsudin@uny.ac.id, harun@uny.ac.id

\begin{tabular}{|c|c|}
\hline ARTICLE INFO & ABSTRACT \\
\hline $\begin{array}{l}\text { Received: } \\
\text { December, 26 } \\
2021 \\
\text { Revised: } \\
\text { January, } 17^{\text {th }} \\
2022 \\
\text { Approved: } \\
\text { January, } 18^{\text {th }} \\
2022\end{array}$ & $\begin{array}{l}\text { The purpose of this study was to describe the practice of art } \\
\text { learning in Kindergarten during the COVID-19 pandemic. } \\
\text { The research method used was a survey. The research } \\
\text { target is that a detailed description of art learning practices } \\
\text { in Kindergarten during the COVID-19 pandemic can be } \\
\text { drawn up as a basis for formulating the basic needs for } \\
\text { improving the quality of learning. The results of the } \\
\text { research can be used to improve educational praxis, } \\
\text { formulate hypotheses, create initial development plans, } \\
\text { evaluate programs, and evaluate policies, especially art } \\
\text { learning in Kindergarten units. The results showed that } \\
\text { PAUD teachers developed an art lesson plan and } \\
\text { implemented it. However, the type of art that was taught } \\
\text { was visual art, while dance and music were never } \\
\text { performed. Likewise, the use of WhatsApp media is more } \\
\text { widely used in online art learning than other social media. } \\
\text { Also using audio media and taking examples of art learning } \\
\text { from social media. Parents actively participate in taking } \\
\text { learning media to school and sending photos and videos of } \\
\text { the learning process at home. With online learning, } \\
\text { teachers have difficulty using technology for early } \\
\text { childhood art learning, and prefer to use face-to-face } \\
\text { learning. Likewise with parents, parents prefer their } \\
\text { children to learn face to face so they can meet the teacher } \\
\text { directly. }\end{array}$ \\
\hline
\end{tabular}

KEYWORDS $\quad$ Art Learning, Kindergarten, Covid 19




\section{INTRODUCTION}

Art is a beauty. It is unavoidable that art is always present in the form of beauty. Art is created for various reasons or reasons according to the human condition that makes it, but a work that is considered to have artistic value usually also contains an aesthetic element. Standards for aesthetic or aesthetic elements have a relative nature, depending on the culture and way of life of humans in a particular area (Gobster, Nassauer, Daniel, \& Fry, 2007). This means that standards for beauty can differ from one community to another (Tang et al., 2012).

The results or processes of human work and ideas that involve skilled, creative abilities, sensory sensitivity, heart sensitivity and thought to produce a work that has a beautiful, harmonious, artistic value and others are usually called art (Rahman, 2019). In the creation or arrangement of a work of art carried out by artists, creative skills are needed specifically according to the type of artwork they make (Burton, Horowitz, \& Abeles, 1999)(Weisberg, 2006)(Niu \& Sternberg, 2001). Studying art is an understanding of aesthetics (beauty) and the re-disclosure of aesthetics in a work of art. Understanding aesthetics is an event of entering aesthetics through the sense of taste and thought to objectify.

Art is fundamental in early childhood education (Prince, 2008). This does not mean to exclude other subject areas. However, it seems that there is no program for children that can succeed without emphasizing the arts. After all, art always makes children feel happy and happy. Why are children always happy? The fact is that art materials stimulate children's creativity in different ways, as Dewey said in (Grube, Delvendahl, Seefeld, \& Volta, 2012) that they make children think. Perhaps that is the reason why children love art.

Art education is essentially a process of human formation through art (Eisner, 1987; Gardner, 1990). Education in general serves to develop the ability of every child to find fulfillment in life, to transmit cultural heritage, to expand social awareness and as a way to increase knowledge. In this case, the goal of art education is actually in line with the broad responsibilities of educational goals in general.

Art education as a form to shape the attitude and personality of children who have mental functions which include fantasy, sensitivity, creativity and expression. A child can fantasize about his work, through the feeling of the child pouring his ideas into the work, making the child sensitive, making the child have good creativity, and expressing the work of art.

Art education is concerned with the beauty of one's creations (Eisner, 2008; Macdonald, 2004). Through experience, children can pour their ideas into works of art. Art education can make the right brain and left brain develop properly.

The development of children's creativity from an early age is very necessary and the role of educators is very important. Efforts to increase creativity can be done by educators. These various efforts refer to the nature of creativity, the role of educators in developing creativity, and efforts to increase children's creativity.

At the beginning of 2020 Indonesia experienced the Covid 19 pandemic, this pandemic was not only felt in Indonesia but throughout the world. This pandemic is something that must be faced together. Community life activities are limited, starting from sports, offices, tourism, and education is no exception. Education, which is very close to interacting with each other, involving many educators and students in the 
learning environment, must undergo changes. In terms of education, which used to be done face-to-face, now it must be done online or online by utilizing internet facilities. Online learning is one solution that inevitably has to be done in order to keep learning going. This online school policy applies to all levels, from PAUD to tertiary institutions.

PAUD institutions which usually do a lot of stimulating development with faceto-face services in the classroom must now be changed in their respective homes. Online learning is a learning design system where the application uses the internet network and is carried out indirectly between teachers and students. Online learning requires all parties to work together in this learning such as parents, teachers and students. Teachers also have to work more creatively and extra in the learning planning process such as preparing materials, materials, methods, and RPPH, as well as evaluations used during the online learning process that are different from before the pandemic.

With social restrictions and physical distancing, the government's ministry of education and culture has banned schools from carrying out face-to-face learning and turning it into online learning. As a teacher, teachers must continue to carry out their obligations as teachers and ensure that children can obtain information and knowledge.

Teachers will certainly try to make what is taught can be understood and understood through various ways, strategies, and methods. But in fact, not a few teachers also experience obstacles in this online learning process. Most teachers have difficulty making learning materials related to aspects of child development including cognitive, language, social emotional, physical motoric, and art development with media in the form of videos/slides/animations either due to inadequate facilities or limited teacher skills. In a study (Novitasari, Prastyo, Iftitah, Reswari, \& Fauziddin, 2021) the organization of materials reached a percentage of $60.36 \%$, this figure shows that there are still many teachers who have not been able to organize materials for online learning. Online learning activities carried out by teachers so far by giving assignments to students. This assignment also applies to art learning. The teacher gives assignments in the form of work-based activities for children.

Therefore, in this survey research, it is intended to find out art learning in kindergartens in the city of Yogyakarta. The results are expected to serve as a basis for formulating the basic needs for improving the quality of learning. The research results can be used to improve educational praxis, formulate hypotheses, create initial development plans, evaluate programs, and evaluate policies, especially art learning in Kindergarten units.

\section{RESEARCH METHOD}

This type of research is a survey. The types of data collected are categorical and numerical data. Categorical data includes demographic data of each kindergarten institution. Numerical data includes the frequency of art learning. The data analysis technique used a descriptive-quantitative approach. The research design includes formulating problems, formulating hypotheses, compiling instrument grids, collecting data, analyzing data, and drawing conclusions. The research population was 235 kindergarten institutions. The sample was 70 kindergarten institutions with a sampling error of 5\% (Tora Yamane, 1967). The sampling technique used proportional random sampling per area of the city of Yogyakarta, Sleman Regency, Bantul Regency, Gunungkidul Regency, Salatiga City, and Kebumen Regency. Data collection techniques used closed questionnaires and open questionnaires. The data analysis technique used a descriptive-quantitative approach for both categorical and numeric data types. 


\section{RESULT AND DISCUSSION}

\section{Early Childhood Art Learning}

Indicators of early childhood art learning include the ability to draw up RPPH for art learning, carry out art lessons, compose learning media, stimulate fine arts, dance, and music.

Table 1. Preparing RPPH for Art Learning

\begin{tabular}{ccccc}
\hline Scale & Frequency & Percent & Valid Percent & Cumulative Percent \\
\hline 1.00 & 27 & 13.1 & 13.1 & 13.1 \\
\hline 2.00 & 32 & 15.5 & 15.5 & 28.6 \\
\hline 3.00 & 33 & 16.0 & 16.0 & 44.7 \\
\hline 4.00 & 35 & 17.0 & 17.0 & 61.7 \\
\hline 5.00 & 79 & 38.3 & 38.3 & 100.0 \\
\hline Total & 206 & 100.0 & 100.0 & \\
\hline
\end{tabular}

The teacher arranges RPPH for art learning with the categories ALWAYS $38.3 \%$, often $17.0 \%$, SOMETIMES as much as $16.0 \%$, seldom as much as $15.5 \%$, and NEVER as much as $13.1 \%$.

Table 2. Art learning services during the pandemic

\begin{tabular}{ccccc}
\hline Scale & Frequency & Percent & Valid Percent & Cumulative Percent \\
\hline 1.00 & 8 & 3.9 & 3.9 & 3.9 \\
\hline 2.00 & 31 & 15.0 & 15.0 & 18.9 \\
\hline 3.00 & 51 & 24.8 & 24.8 & 43.7 \\
\hline 4.00 & 57 & 27.7 & 27.7 & 71.4 \\
\hline 5.00 & 59 & 28.6 & 28.6 & 100.0 \\
\hline Total & 206 & 100.0 & 100.0 & \\
\hline
\end{tabular}

The teacher carries out art learning in the categories of ALWAYS as much as $28.6 \%$, often as much as $27.7 \%$, SOMETIMES as much as $24.8 \%$, seldom as much as $15.0 \%$, and NEVER as much as $3.9 \%$.

Table 3. Art learning media

\begin{tabular}{ccccc}
\hline Scale & Frequency & Percent & Valid Percent & Cumulative Percent \\
\hline 1.00 & 8 & 3.9 & 3.9 & 3.9 \\
\hline 2.00 & 32 & 15.5 & 15.5 & 19.4 \\
\hline 3.00 & 50 & 24.3 & 24.3 & 43.7 \\
\hline 4.00 & 60 & 29.1 & 29.1 & 72.8 \\
\hline 5.00 & 56 & 27.2 & 27.2 & 100.0 \\
\hline Total & 206 & 100.0 & 100.0 & \\
\hline
\end{tabular}

The teacher makes art learning media with the categories of ALWAYS as much as $27.2 \%$, often as much as $29.1 \%$, SOMETIMES as much as $24.3 \%$, seldom as much as $15.5 \%$, and NEVER as much as $3.9 \%$.

Table 4. Fine arts lessons

\begin{tabular}{ccccc}
\hline Skala & Frequency & Percent & Valid Percent & Cumulative Percent \\
\hline 1.00 & 22 & 10.7 & 10.7 & 10.7 \\
\hline 2.00 & 46 & 22.3 & 22.3 & 33.0 \\
\hline 3.00 & 66 & 32.0 & 32.0 & 65.0 \\
\hline 4.00 & 46 & 22.3 & 22.3 & 87.4 \\
\hline
\end{tabular}




\begin{tabular}{ccccc}
\hline 5.00 & 26 & 12.6 & 12.6 & 100.0 \\
\hline Total & 206 & 100.0 & 100.0 & \\
\hline
\end{tabular}

The teacher carries out art learning in the categories of ALWAYS as much as $12.6 \%$, often as much as $22.3 \%$, SOMETIMES as much as $32.0 \%$, seldom as much as $22.3 \%$, and NEVER as much as $10.7 \%$.

Table 5. Dance lessons

\begin{tabular}{ccccc}
\hline Skala & Frequency & Percent & Valid Percent & Cumulative Percent \\
\hline 1.00 & 103 & 50.0 & 50.0 & 50.0 \\
\hline 2.00 & 43 & 20.9 & 20.9 & 70.9 \\
\hline 3.00 & 42 & 20.4 & 20.4 & 91.3 \\
\hline 4.00 & 12 & 5.8 & 5.8 & 97.1 \\
\hline 5.00 & 6 & 2.9 & 2.9 & 100.0 \\
\hline Total & 206 & 100.0 & 100.0 & \\
\hline
\end{tabular}

The teacher carries out dance lessons in the categories of ALWAYS as much as $2.9 \%$, often as much as $5.8 \%$, SOMETIMES as much as $20.4 \%$, seldom as much as $20.9 \%$, and NEVER as much as $50.0 \%$.

Table 6. Music art learning

\begin{tabular}{ccccc}
\hline Skala & Frequency & Percent & Valid Percent & Cumulative Percent \\
\hline 1.00 & 59 & 28.6 & 28.6 & 28.6 \\
\hline 2.00 & 57 & 27.7 & 27.7 & 56.3 \\
\hline 3.00 & 54 & 26.2 & 26.2 & 82.5 \\
\hline 4.00 & 24 & 11.7 & 11.7 & 94.2 \\
\hline 5.00 & 12 & 5.8 & 5.8 & 100.0 \\
\hline Total & 206 & 100.0 & 100.0 & \\
\hline
\end{tabular}

The teacher carries out music learning in the categories ALWAYS as much as $5.8 \%$, often as much as $11.7 \%$, SOMETIMES as much as $26.2 \%$, seldom as much as $27.7 \%$, and NEVER as much as $28.6 \%$.

Conclusion: PAUD teachers prepare art lesson plans and implement them. However, the type of art that was taught was visual art, while dance and music were never performed.

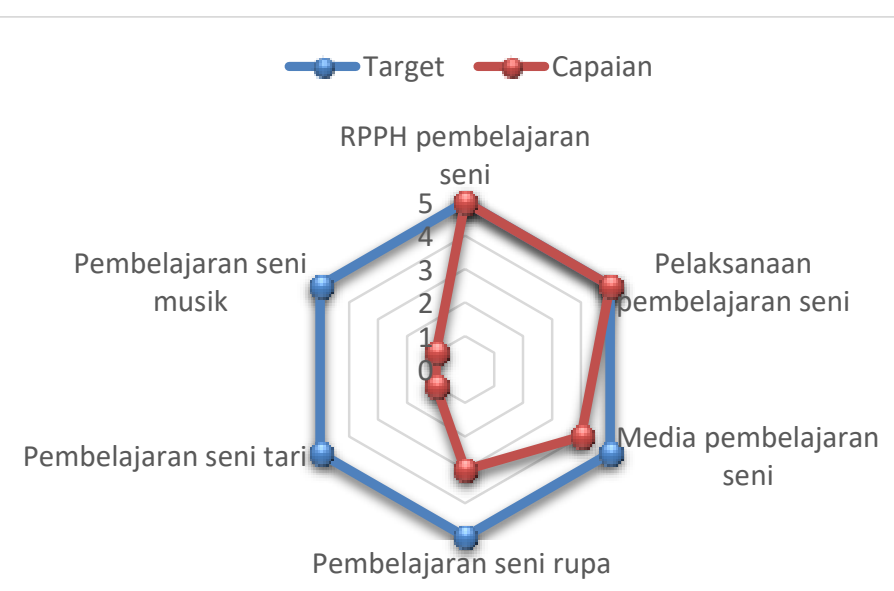

Figure 1. Art Planning \& Learning 


\section{Art Teaching Materials}

Art learning media used during the Covid-19 pandemic include WhatsApp, Google Meet, Google Classroom, Zoom Meeting, MS Teams, Audio, and Youtube.

Table 7. Creating art learning media

\begin{tabular}{ccccc}
\hline Scale & Frequency & Percent & Valid Percent & Cumulative Percent \\
\hline 1.00 & 57 & 27.7 & 27.7 & 27.7 \\
\hline 2.00 & 55 & 26.7 & 26.7 & 54.4 \\
\hline 3.00 & 49 & 23.8 & 23.8 & 78.2 \\
\hline 4.00 & 27 & 13.1 & 13.1 & 91.3 \\
\hline 5.00 & 18 & 8.7 & 8.7 & 100.0 \\
\hline Total & 206 & 100.0 & 100.0 & \\
\hline
\end{tabular}

The teacher makes art learning media with the categories ALWAYS as much as $8.7 \%$, often as much as $13.1 \%$, SOMETIMES as much as $23.8 \%$, seldom as much as $26.7 \%$, and NEVER as much as $27.7 \%$.

Table 8. Provide examples of art learning videos from social media

\begin{tabular}{ccccc}
\hline Scale & Frequency & Percent & Valid Percent & Cumulative Percent \\
\hline 1.00 & 54 & 26.2 & 26.2 & 26.2 \\
\hline 2.00 & 54 & 26.2 & 26.2 & 52.4 \\
\hline 3.00 & 58 & 28.2 & 28.2 & 80.6 \\
\hline 4.00 & 22 & 10.7 & 10.7 & 91.3 \\
\hline 5.00 & 18 & 8.7 & 8.7 & 100.0 \\
\hline Total & 206 & 100.0 & 100.0 & \\
\hline
\end{tabular}

The teacher gives examples of art learning videos from social media with the categories ALWAYS 8.7\%, OFTEN as much as 10.7\%, SOMETIMES as much as $28.2 \%$, seldom as much as $26.2 \%$, and NEVER as much as $26.2 \%$.

Table 9. Children's Worksheet Media

\begin{tabular}{ccccc}
\hline Scale & Frequency & Percent & Valid Percent & Cumulative Percent \\
\hline 1.00 & 33 & 16.0 & 16.0 & 16.0 \\
\hline 2.00 & 50 & 24.3 & 24.3 & 40.3 \\
\hline 3.00 & 53 & 25.7 & 25.7 & 66.0 \\
\hline 4.00 & 52 & 25.2 & 25.2 & 91.3 \\
\hline 5.00 & 18 & 8.7 & 8.7 & 100.0 \\
\hline Total & 206 & 100.0 & 100.0 & \\
\hline
\end{tabular}

The teacher uses children's worksheets as an art learning medium with the categories ALWAYS 8.7\%, often as much as 25.2\%, SOMETIMES as much as $25.7 \%$, rarely as much as $24.3 \%$, and NEVER as much as $16.0 \%$.

Table 10. Audio Media

\begin{tabular}{ccccc}
\hline Scale & Frequency & Percent & Valid Percent & Cumulative Percent \\
\hline 1.00 & 38 & 18.4 & 18.4 & 18.4 \\
\hline 2.00 & 51 & 24.8 & 24.8 & 43.2 \\
\hline 3.00 & 58 & 28.2 & 28.2 & 71.4 \\
\hline 4.00 & 44 & 21.4 & 21.4 & 92.7 \\
\hline 5.00 & 15 & 7.3 & 7.3 & 100.0 \\
\hline Total & 206 & 100.0 & 100.0 & \\
\hline
\end{tabular}


The teacher uses children's worksheets as art learning media with the categories ALWAYS as much as $7.3 \%$, often as much as $21.4 \%$, SOMETIMES as much as $28.2 \%$, seldom as much as $24.8 \%$, and NEVER as much as $18.4 \%$.

Table 11. WhatsApp Media

\begin{tabular}{ccccc}
\hline Scale & Frequency & Percent & Valid Percent & Cumulative Percent \\
\hline 1.00 & 26 & 12.6 & 12.6 & 12.6 \\
\hline 2.00 & 34 & 16.5 & 16.5 & 29.1 \\
\hline 3.00 & 44 & 21.4 & 21.4 & 50.5 \\
\hline 4.00 & 52 & 25.2 & 25.2 & 75.7 \\
\hline 5.00 & 50 & 24.3 & 24.3 & 100.0 \\
\hline Total & 206 & 100.0 & 100.0 & \\
\hline
\end{tabular}

Teachers use WhatsApp as an art learning medium with the categories ALWAYS $24.3 \%$, often as much as $25.2 \%$, SOMETIMES as much as $21.4 \%$, seldom as much as $16.5 \%$, and NEVER as much as $12.6 \%$.

Table 12 Frequency of WAG Usage

\begin{tabular}{ccccc}
\hline Scale & Frequency & Percent & Valid Percent & Cumulative Percent \\
\hline 1.00 & 30 & 14.6 & 14.6 & 14.6 \\
\hline 2.00 & 43 & 20.9 & 20.9 & 35.4 \\
\hline 3.00 & 45 & 21.8 & 21.8 & 57.3 \\
\hline 4.00 & 47 & 22.8 & 22.8 & 80.1 \\
\hline 5.00 & 41 & 19.9 & 19.9 & 100.0 \\
\hline Total & 206 & 100.0 & 100.0 & \\
\hline
\end{tabular}

The frequency of teachers using WhatsApp as an art learning medium with the categories ALWAYS as much as $19.9 \%$, often as much as $22.8 \%$, sometimes as much as $21.8 \%$, rarely as much as $20.9 \%$, and NEVER as much as $14.6 \%$.

Table 13. Media Zoom Meeting

\begin{tabular}{ccccc}
\hline Scale & Frequency & Percent & Valid Percent & Cumulative Percent \\
\hline 1.00 & 150 & 72.8 & 72.8 & 72.8 \\
\hline 2.00 & 21 & 10.2 & 10.2 & 83.0 \\
\hline 3.00 & 19 & 9.2 & 9.2 & 92.2 \\
\hline 4.00 & 11 & 5.3 & 5.3 & 97.6 \\
\hline 5.00 & 5 & 2.4 & 2.4 & 100.0 \\
\hline Total & 206 & 100.0 & 100.0 & \\
\hline
\end{tabular}

The teacher uses Zoom Meeting as an art learning medium with the categories ALWAYS as much as 2.4\%, often as much as 5.3\%, SOMETIMES as much as $9.2 \%$, seldom as much as $10.2 \%$, and NEVER as much as $72.8 \%$.

Table 14. Frequency of Use of Zoom Meetings

\begin{tabular}{ccccc}
\hline Scale & Frequency & Percent & Valid Percent & Cumulative Percent \\
\hline 1.00 & 154 & 74.8 & 74.8 & 74.8 \\
\hline 2.00 & 19 & 9.2 & 9.2 & 84.0 \\
\hline 3.00 & 20 & 9.7 & 9.7 & 93.7 \\
\hline 4.00 & 8 & 3.9 & 3.9 & 97.6 \\
\hline 5.00 & 5 & 2.4 & 2.4 & 100.0 \\
\hline Total & 206 & 100.0 & 100.0 & \\
\hline
\end{tabular}




\section{Joko Pamungkas, Amir Syamsudin, Harun Ar Rosid}

The frequency of teachers using Zoom Meetings as an art learning medium with the categories ALWAYS as much as $2.4 \%$, often as much as $3.98 \%$, SOMETIMES as much as $9.7 \%$, seldom as much as $9.2 \%$, and NEVER as much as $74.8 \%$.

Table 15. Media Google Meet

\begin{tabular}{ccccc}
\hline Scale & Frequency & Percent & Valid Percent & Cumulative Percent \\
\hline 1.00 & 168 & 81.6 & 81.6 & 81.6 \\
\hline 2.00 & 16 & 7.8 & 7.8 & 89.3 \\
\hline 3.00 & 14 & 6.8 & 6.8 & 96.1 \\
\hline 4.00 & 5 & 2.4 & 2.4 & 98.5 \\
\hline 5.00 & 3 & 1.5 & 1.5 & 100.0 \\
\hline Total & 206 & 100.0 & 100.0 & \\
\hline
\end{tabular}

Teachers use Google Meetings as an art learning medium with the categories ALWAYS $1.54 \%$, OFTEN as much as $2.4 \%$, SOMETIMES as much as $6.8 \%$, seldom as much as $7.8 \%$, and NEVER as much as $81.6 \%$.

Table 16. Frequency of Use of Google Meet

\begin{tabular}{ccccc}
\hline Scale & Frequency & Percent & Valid Percent & Cumulative Percent \\
\hline 1.00 & 167 & 81.1 & 81.1 & 81.1 \\
\hline 2.00 & 19 & 9.2 & 9.2 & 90.3 \\
\hline 3.00 & 13 & 6.3 & 6.3 & 96.6 \\
\hline 4.00 & 5 & 2.4 & 2.4 & 99.0 \\
\hline 5.00 & 2 & 1.0 & 1.0 & 100.0 \\
\hline Total & 206 & 100.0 & 100.0 & \\
\hline
\end{tabular}

The frequency with which teachers use Google Meetings as an art learning medium is ALWAYS 1.0\%, often 2.4\%, sometimes 6.3\%, rarely 9.2\%, and NEVER $81.1 \%$.

Table 17. MS Teams Media

\begin{tabular}{ccccc}
\hline Scale & Frequency & Percent & Valid Percent & Cumulative Percent \\
\hline 1.00 & 171 & 83.0 & 83.0 & 83.0 \\
\hline 2.00 & 18 & 8.7 & 8.7 & 91.7 \\
\hline 3.00 & 13 & 6.3 & 6.3 & 98.1 \\
\hline 4.00 & 3 & 1.5 & 1.5 & 99.5 \\
\hline 5.00 & 1 & .5 & .5 & 100.0 \\
\hline Total & 206 & 100.0 & 100.0 & \\
\hline
\end{tabular}

The teacher uses MS Teams as an art learning medium with the categories ALWAYS 0.5\%, FREQUENTLY 1.5\%, SOMETIMES 6.3\%, RARELY 8.7\%, and NEVER $83.0 \%$.

Table 18. Frequency of Using MS Teams

\begin{tabular}{ccccc}
\hline Scale & Frequency & Percent & Valid Percent & Cumulative Percent \\
\hline 1.00 & 172 & 83.5 & 83.5 & 83.5 \\
\hline 2.00 & 16 & 7.8 & 7.8 & 91.3 \\
\hline 3.00 & 11 & 5.3 & 5.3 & 96.6 \\
\hline 4.00 & 3 & 1.5 & 1.5 & 98.1 \\
\hline 5.00 & 4 & 1.9 & 1.9 & 100.0 \\
\hline Total & 206 & 100.0 & 100.0 & \\
\hline
\end{tabular}


The frequency of teachers using MS Teams as an art learning medium with the categories ALWAYS 1.0\%, FREQUENTLY 2.4\%, SOMETIMES as much as $6.3 \%$, rarely $9.2 \%$, and NEVER as much as $81.1 \%$.

Table 19. Google Classroom

\begin{tabular}{ccccc}
\hline Scale & Frequency & Percent & Valid Percent & Cumulative Percent \\
\hline 1.00 & 185 & 89.8 & 89.8 & 89.8 \\
\hline 2.00 & 11 & 5.3 & 5.3 & 95.1 \\
\hline 3.00 & 9 & 4.4 & 4.4 & 99.5 \\
\hline 5.00 & 1 & .5 & .5 & 100.0 \\
\hline Total & 206 & 100.0 & 100.0 & \\
\hline
\end{tabular}

Teachers use Google Classroom as an art learning medium with the categories ALWAYS $0.5 \%$, FREQUENTLY $0.0 \%$, SOMETIMES as much as $4.4 \%$, RARELY as much as $5.3 \%$, and NEVER as much as $89.8 \%$.

Table 20. Google Classroom Frequency

\begin{tabular}{ccccc}
\hline Scale & Frequency & Percent & Valid Percent & Cumulative Percent \\
\hline 1.00 & 184 & 89.3 & 89.3 & 89.3 \\
\hline 2.00 & 12 & 5.8 & 5.8 & 95.1 \\
\hline 3.00 & 7 & 3.4 & 3.4 & 98.5 \\
\hline 4.00 & 2 & 1.0 & 1.0 & 99.5 \\
\hline 5.00 & 1 & .5 & .5 & 100.0 \\
\hline Total & 206 & 100.0 & 100.0 & \\
\hline
\end{tabular}

The frequency of teachers using Google Classroom as an art learning medium with the categories ALWAYS 0.5\%, FREQUENTLY 1.0\%, SOMETIMES as much as 3.4\%, RARELY $5.2 \%$, and NEVER as much as $89.3 \%$.

Table 21. Youtube Media

\begin{tabular}{ccccc}
\hline Scale & Frequency & Percent & Valid Percent & Cumulative Percent \\
\hline 1.00 & 95 & 46.1 & 46.1 & 46.1 \\
\hline 2.00 & 42 & 20.4 & 20.4 & 66.5 \\
\hline 3.00 & 45 & 21.8 & 21.8 & 88.3 \\
\hline 4.00 & 16 & 7.8 & 7.8 & 96.1 \\
\hline 5.00 & 8 & 3.9 & 3.9 & 100.0 \\
\hline Total & 206 & 100.0 & 100.0 & \\
\hline
\end{tabular}

Teachers use YouTube as an art learning medium with 3.9\% ALWAYS, 7.8\% OFTEN, 21.8\% SOMETIMES, 20.4\% RARE, and 46.1\% NEVER.

Table 22. Youtube Usage Frequency

\begin{tabular}{ccccc}
\hline Scale & Frequency & Percent & Valid Percent & Cumulative Percent \\
\hline 1.00 & 107 & 51.9 & 51.9 & 51.9 \\
\hline 2.00 & 42 & 20.4 & 20.4 & 72.3 \\
\hline 3.00 & 38 & 18.4 & 18.4 & 90.8 \\
\hline 4.00 & 13 & 6.3 & 6.3 & 97.1 \\
\hline 5.00 & 6 & 2.9 & 2.9 & 100.0 \\
\hline Total & 206 & 100.0 & 100.0 & \\
\hline
\end{tabular}

The frequency of teachers using Youtube as an art learning medium with the categories ALWAYS as much as $2.9 \%$, often as much as 6.3\%, SOMETIMES as much as 
$18.4 \%$, seldom as much as $20.4 \%$, and NEVER as much as $51.9 \%$.

Conclusion: PAUD teachers use WhatsAPP more in online art learning than other social media. Also using audio media and taking examples of art learning from social media.

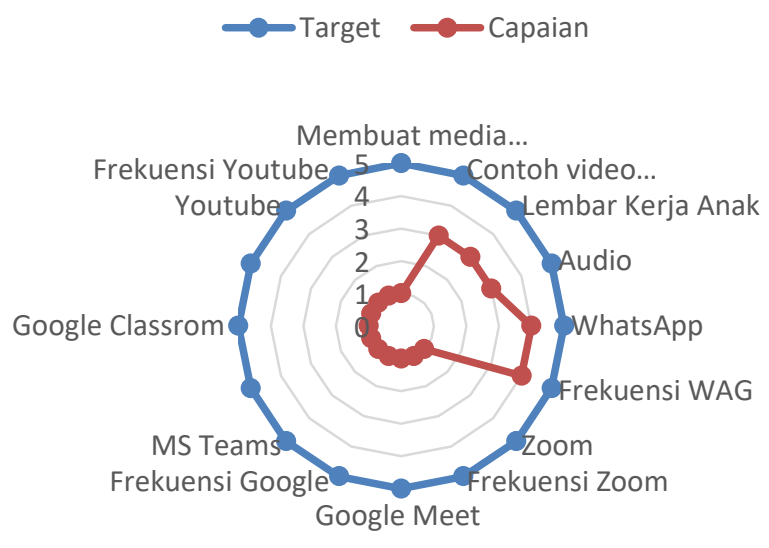

Figure 2. Online Media for Learning Arts

\section{Parental Involvement}

The involvement of parents in online learning includes taking learning materials to school, sending photos or videos of children's art learning processes at home, difficulties faced by parents in using technology for art learning, and offline learning assistance.

Table 23. Parents Take Learning Media

\begin{tabular}{ccccc}
\hline Scale & Frequency & Percent & Valid Percent & Cumulative Percent \\
\hline 1.00 & 18 & 8.7 & 8.7 & 8.7 \\
\hline 2.00 & 47 & 22.8 & 22.8 & 31.6 \\
\hline 3.00 & 44 & 21.4 & 21.4 & 52.9 \\
\hline 4.00 & 46 & 22.3 & 22.3 & 75.2 \\
\hline 5.00 & 51 & 24.8 & 24.8 & 100.0 \\
\hline Total & 206 & 100.0 & 100.0 & \\
\hline
\end{tabular}

Parental participation in the form of willingness to take art learning media to school can be grouped into categories ALWAYS as much as $24.8 \%$, often as much as $22.3 \%$, SOMETIMES as much as $21.4 \%$, rarely as much as $22.8 \%$, and NEVER as much as $8.7 \%$.

Table 24. Photos and videos of evidence of art learning

\begin{tabular}{ccccc}
\hline Scale & Frequency & Percent & Valid Percent & Cumulative Percent \\
\hline 1.00 & 8 & 3.9 & 3.9 & 3.9 \\
\hline 2.00 & 26 & 12.6 & 12.6 & 16.5 \\
\hline 3.00 & 27 & 13.1 & 13.1 & 29.6 \\
\hline 4.00 & 58 & 28.2 & 28.2 & 57.8 \\
\hline 5.00 & 87 & 42.2 & 42.2 & 100.0 \\
\hline Total & 206 & 100.0 & 100.0 & \\
\hline
\end{tabular}

Parental participation in the form of sending photos and videos of the results of art learning to schools can be grouped into categories ALWAYS 42.2\%, FREQUENTLY $28.2 \%$, SOMETIMES as much as $13.1 \%$, Rarely as much as $12.6 \%$, and NEVER as much as $3.9 \%$. 
Table 25. Difficulties in designing online art lessons

\begin{tabular}{ccccc}
\hline Scale & Frequency & Percent & Valid Percent & Cumulative Percent \\
\hline 1.00 & 15 & 7.3 & 7.3 & 7.3 \\
\hline 2.00 & 41 & 19.9 & 19.9 & 27.2 \\
\hline 3.00 & 106 & 51.5 & 51.5 & 78.6 \\
\hline 4.00 & 33 & 16.0 & 16.0 & 94.7 \\
\hline 5.00 & 11 & 5.3 & 5.3 & 100.0 \\
\hline Total & 206 & 100.0 & 100.0 & \\
\hline
\end{tabular}

Parents' difficulties in designing art learning via online can be grouped into categories ALWAYS as much as 5.3\%, often as much as $16.03 \%$, SOMETIMES as much as $51.5 \%$, seldom as much as $19.9 \%$, and NEVER as much as $7.3 \%$.

Table 26. Difficulties in using technology for art learning

\begin{tabular}{ccccc}
\hline Scale & Frequency & Percent & Valid Percent & Cumulative Percent \\
\hline 1.00 & 16 & 7.8 & 7.8 & 7.8 \\
\hline 2.00 & 42 & 20.4 & 20.4 & 28.2 \\
\hline 3.00 & 92 & 44.7 & 44.7 & 72.8 \\
\hline 4.00 & 45 & 21.8 & 21.8 & 94.7 \\
\hline 5.00 & 11 & 5.3 & 5.3 & 100.0 \\
\hline Total & 206 & 100.0 & 100.0 & \\
\hline
\end{tabular}

Difficulties in using technology for art learning can be grouped into categories ALWAYS as much as $5.3 \%$, often as much as $21.8 \%$, SOMETIME as much as $44.7 \%$, rarely as much as $20.4 \%$, and NEVER as much as $7.8 \%$.

Table 27. Not sending proof of art learning

\begin{tabular}{ccccc}
\hline Scale & Frequency & Percent & Valid Percent & Cumulative Percent \\
\hline 1.00 & 18 & 8.7 & 8.7 & 8.7 \\
\hline 2.00 & 48 & 23.3 & 23.3 & 32.0 \\
\hline 3.00 & 92 & 44.7 & 44.7 & 76.7 \\
\hline 4.00 & 39 & 18.9 & 18.9 & 95.6 \\
\hline 5.00 & 9 & 4.4 & 4.4 & 100.0 \\
\hline Total & 206 & 100.0 & 100.0 & \\
\hline
\end{tabular}

Parents who do not send proof of art learning can be grouped into categories ALWAYS as much as $4.4 \%$, often as much as $18.9 \%$, SOMETIMES as much as $44.7 \%$, seldom as much as $23.3 \%$, and NEVER as much as $8.7 \%$.

Table 28. Learning art offline

\begin{tabular}{ccccc}
\hline Scale & Frequency & Percent & Valid Percent & Cumulative Percent \\
\hline 1.00 & 22 & 10.7 & 10.7 & 10.7 \\
\hline 2.00 & 39 & 18.9 & 18.9 & 29.6 \\
\hline 3.00 & 56 & 27.2 & 27.2 & 56.8 \\
\hline 4.00 & 56 & 27.2 & 27.2 & 84.0 \\
\hline 5.00 & 33 & 16.0 & 16.0 & 100.0 \\
\hline Total & 206 & 100.0 & 100.0 & \\
\hline
\end{tabular}

Offline art learning can be grouped into categories ALWAYS as much as $16.0 \%$, often as much as $27.2 \%$, SOMETIME as much as $27.2 \%$, seldom as much as $18.9 \%$, and NEVER as much as $10.7 \%$. 


\section{Joko Pamungkas, Amir Syamsudin, Harun Ar Rosid}

Conclusion: Parents actively participate in taking learning media to school and sending photos and videos of the learning process at home. Teachers have difficulty using technology for early childhood art learning, and prefer to use face-to-face learning.

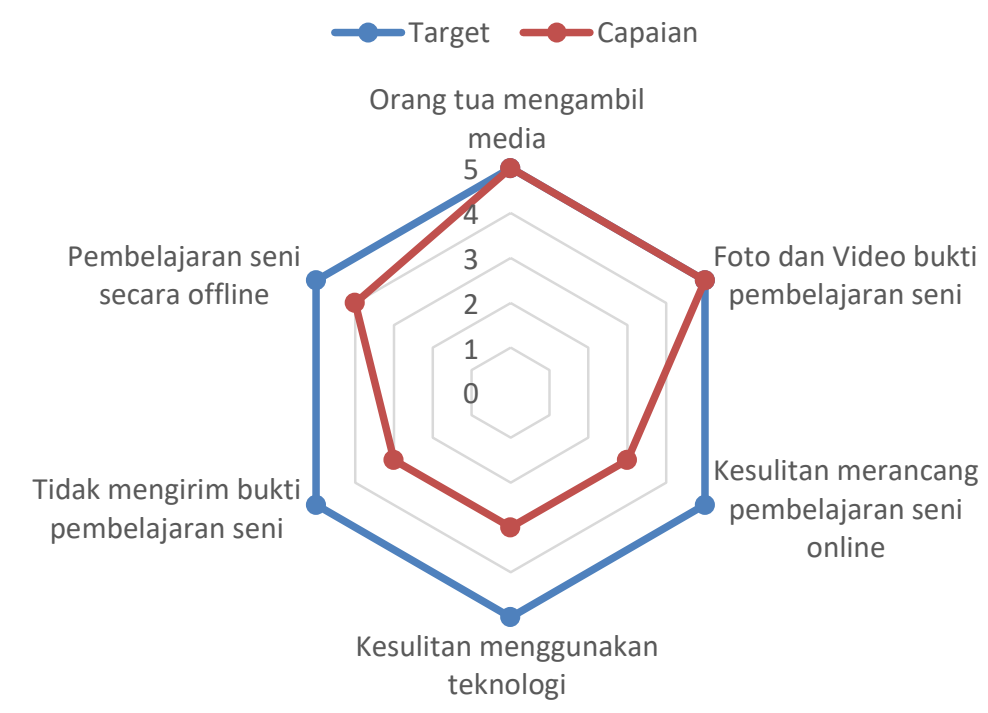

Figure 3. Parental Participation in Art Learning

Based on the qualitative analysis of Atlas.Ti, it was found that the assessment of art learning carried out by teachers was the result of work (166), observation (76), and performance (75). Parents of children prefer face-to-face learning rather than face-to-face. The reason is that face-to-face learning is more varied, flexible, and children are taught directly by the teacher, while face-to-face learning is constrained by the operation of information technology, networks that are not strong, and cannot optimally accompany their children. The hope in the new normal era is for children to return to school and carry out activities like pre-covid: free to do activities in public places and face-to-face learning. Art lessons in the new normal era that will be carried out by teachers are dance, music, and fine arts.

\section{CONCLUSION}

Teachers plan art lessons, create media, and implement them during the pandemic. However, the focus of learning is only on visual arts, while dance and music are not carried out. The online learning mode most often used by teachers and parents of children during the pandemic is WhatsApp. Meanwhile, audio media and children's worksheets are sometimes used. The practice of learning art during the pandemic is for parents to come to school to take LKA and the children do it in their respective homes. The results of the children's work are returned by the parents to the school when they take the next LKA assignment. The obstacle for the majority of parents in online learning is that they cannot operate communication tools such as mobile phones for video conferences, or the internet network is weak, or they do not have the tools to go online. 


\section{REFERENCES}

Burton, Judith, Horowitz, Robert, \& Abeles, Hal. (1999). Learning in and through the arts: Curriculum implications. Champions of Change: The Impact of the Arts on Learning, 35-46.

Eisner, Elliot W. (1987). The role of discipline-based art education in America's schools. Art Education, 40(5), 6-45.

Eisner, Elliot W. (2008). The arts and the creation of mind. Yale University Press.

Gardner, Howard. (1990). Art education and human development (Vol. 3). Getty Publications.

Gobster, Paul H., Nassauer, Joan I., Daniel, Terry C., \& Fry, Gary. (2007). The shared landscape: what does aesthetics have to do with ecology? Landscape Ecology, 22(7), 959-972.

Grube, Nikolai, Delvendahl, Kai, Seefeld, Nicolaus, \& Volta, Beniamino. (2012). Under the Rule of the Snake Kings: Uxul in the 7th and 8th Centuries. Estudios de Cultura Maya, 40, 11-49.

Macdonald, Stuart. (2004). The history and philosophy of art education. James Clarke \& Co.

Niu, Weihua, \& Sternberg, Robert J. (2001). Cultural influences on artistic creativity and its evaluation. International Journal of Psychology, 36(4), 225-241.

Novitasari, Yesi, Prastyo, Danang, Iftitah, Selfi Lailiyatul, Reswari, Ardhana, \& Fauziddin, Mohammad. (2021). Media Daur Ulang (Recycle System) dalam Kemampuan Membaca Bahasa Inggris Awal Anak Usia Dini. Jurnal Obsesi: Jurnal Pendidikan Anak Usia Dini, 6(3), 1323-1330.

Prince, Eileen S. (2008). Art is fundamental: Teaching the elements and principles of art in elementary school. Chicago Review Press.

Rahman, Md. (2019). 21st century skill'problem solving': Defining the concept. Rahman, MM (2019). 21st Century Skill "Problem Solving": Defining the Concept. Asian Journal of Interdisciplinary Research, 2(1), 64-74.

Tang, Haifeng, Walsh, Shawn P., Yan, Yan, de Jesus, Reynalda K., Shahripour, Aurash, Teumelsan, Nardos, Zhu, Yuping, Ha, Sookhee, Owens, Karen A., \& ThomasFowlkes, Brande S. (2012). Discovery of selective small molecule ROMK inhibitors as potential new mechanism diuretics. ACS Medicinal Chemistry Letters, 3(5), 367372.

Weisberg, Robert W. (2006). Creativity: Understanding innovation in problem solving, science, invention, and the arts. John Wiley \& Sons. 